\title{
Effect of disease-modifying treatments on antibody-mediated response to anti-COVID19 vaccination in people with multiple sclerosis
}

\author{
Alice Mariottini ${ }^{1,2} \cdot$ Andrea Bertozzi $^{1} \cdot$ Leonardo Marchi $^{1} \cdot$ Maria Di Cristinzi $^{1} \cdot$ Claudia Mechi $^{2}$ - Alessandro Barilaro ${ }^{2}$. \\ Luca Massacesi $^{1,2} \cdot$ Anna Maria Repice ${ }^{2}$
}

Received: 23 November 2021 / Revised: 29 January 2022 / Accepted: 30 January 2022 / Published online: 3 March 2022

(c) The Author(s), under exclusive licence to Springer-Verlag GmbH Germany 2022

\begin{abstract}
Background Few data are available so far on the antibody-mediated immune response to anti-SARS-Cov2 vaccination in people with multiple sclerosis (pwMS) treated with disease-modifying treatments (DMTs), therefore this issue was explored in a real-life cohort of pwMS.

Materials and methods Retrospective monocentric study on anti-spike protein antibody response in pwMS who had received vaccination for Sars-Cov2. Adverse events following vaccination were also recorded.

Results One hundred and twenty pwMS were included: 83 females (69\%); median age at vaccination 42 years (range $21-73) ; 112 / 120$ patients (93\%) were receiving DMTs at vaccination. Anti-spike protein IgG antibodies were detectable in 102/120 (85\%) cases overall, being the proportion lower in pwMS receiving anti-CD20 antibodies (14/31, 45\%) compared to non-depletive treatments $(77 / 78,99 \%), p<0.0001$. Median anti-spike titre was lower in anti-CD20 antibodies and fingolimod-treated pwMS compared to those receiving other DMTs, and it correlated with anti-CD20 treatment duration $(R-0.93, p<0.0001)$ and with age at vaccination in pwMS not receiving depletive treatments $(R-0.25, p=0.028)$. Baseline $\mathrm{CD} 19+$ cell count (where available) was higher in the responder group than in non-responders, $p<0.0001$. Two symptomatic COVID-19 infections were diagnosed over a median follow-up of 5 months (range 2-7); adverse events were aligned with the published literature.

Conclusion Antibody response to anti-COVID-19 vaccines was detected in most of the pwMS analysed, but frequency of responders was reduced in those receiving CD20 depleting therapies compared to other DMTs-treated pwMS. Investigations on cell-mediated immune response are needed to assess whether a protective immune response is elicited also in nonantibody responders.
\end{abstract}

Keywords Multiple sclerosis · COVID-19 vaccines $\cdot$ Disease-modifying therapies $\cdot$ CD20 deplething therapies $\cdot$ Antibody response

The present work was carried out at Azienda OspedalieroUniversitaria Careggi, Largo Brambilla 3, 50134, Florence, Italy.

Anna Maria Repice

repicea@gmail.com

1 Department of Neurosciences, Drug and Child Health, University of Florence, Florence, Italy

2 Department of Neurology 2 and Tuscan Region Multiple Sclerosis Referral Centre, Careggi University Hospital, Florence, Italy

\section{Introduction}

Since March 2020, a viral pandemic is ongoing due to the outbreak of a coronavirus-associated acute respiratory disease called coronavirus disease 19 (COVID-19), caused by the spillover of an animal coronavirus to humans, designated as SARS-Cov2 [1]. COVID-19 is air-transmitted and highly contagious; even if SARS-Cov2 infection is asymptomatic or paucisymptomatic in most of the cases, severe and potentially fatal disease with interstitial pneumonia requiring ventilatory support and intensive care treatment is observed in a not negligible proportion of the cases, mostly those affected by chronic diseases [2]. Multiple sclerosis (MS) is a chronic inflammatory demyelinating autoimmune disease 
of the central nervous system that can lead to physical and cognitive disability [3]. Different disease-modifying treatments (DMTs) are currently approved for the treatment of MS, with immunomodulant/immunosuppressive properties [4]. A possible increase in the risk of severe COVID-19 was previously suggested, mostly associated with old age and severe disability [5]. Moreover, the effect of immunosuppressive or depletive DMTs was suggested to potentially be associated with an increased risk of severe infection, but no definite evidence on this issue is available so far $[6,7]$.

Since December 2020, vaccines with a different mechanism of action (mRNA, DNA, viral vector) were approved for the prevention of SARS-CoV-2 infection $[8,9]$ and people affected by MS were included by the Italian National Institute of Health in a priority class of vaccination, being considered as "category with frailty". Data on immunological response to SARS-Cov2 vaccines are mostly available on healthy individuals and people who were previously infected by the virus [10], and limited data are available so far on people with MS [12].

The aim of the present study is therefore to explore the antibody response to anti-SARS-Cov2 vaccines in a real-life cohort of treated and untreated MS patients.

\section{Materials and methods}

\section{Study design}

A retrospective monocentric study aimed at exploring the antibody response to SARS-Cov2 vaccines in people affected by MS who attended the Tuscan Region MS Referral Centre of the Careggi University Hospital in Florence, Italy.

\section{Patient selection}

MS patients diagnosed according to the Poser (1983) [10] and McDonald criteria [11] who had received the antiSARS-Cov2 vaccines and who underwent serological testing for SARS-Cov2 neutralizing antibodies, i.e., anti-Spike protein (anti-S) at least 1 month following the completion of the vaccination cycle were included.

\section{Clinical examinations and outcomes}

According to the local regulations, patients received two doses of the Comirnaty (BioNTech/Pfizer), or the COVID-19 Vaccine (Moderna), or the COVID-19 vaccine AstraZeneca (Vaxzevria Vaccine), except for those previously infected by SARS-Cov2 who received a single dose of vaccine. Patients regularly attended the MS clinic for neurological followup and/or for the administration of IV DMTs; according to clinical practice, period blood tests for treatment monitoring were undertaken, including total white blood cell count (WBC), lymphocytic count (LC), and immunophenotyping. Assessment performed before receiving the first dose of vaccine was considered as baseline (or T0); T1 assessments were undertaken between the first and the second dose, whereas $\mathrm{T} 2$ assessment (post-vaccine) between 4 and 8 weeks following the completion of the vaccination cycle.

Antibody response was measured in peripheral blood samples by clinical analysis laboratories, and antibody titres below the lower detection cut-off according to the manufacturer's instructions were recorded as 0 .

Clinical-demographic information including age, gender, MS form, disease duration, treatment duration, disability (assessed as Expanded Disability Status Scale, EDSS) [13] was retrospectively collected from clinical records.

Presence (and titre) of SARS-Cov2 neutralizing (anti-S) antibody response detected following the completion of the vaccine cycle were evaluated. Anti-S antibody testing was performed in accredited medical laboratories with electrochemiluminescence immunoassay (ECLIA)-based methods; antibody titres were expressed in binding antibody unit $\mathrm{mL}$ (BAU/mL). In the absence of a cut-off titre of antibodies correlated with a protective antibody response, patients were defined as responders if antibody titre was above the lower detection cut-off according to the manufacturer's instructions, and as non-responders if they had a titre below the cut-off. Correlations between antibody response and baseline characteristics of the patients were explored, including treatment status and the type of DMTs received. According to the national prescribing indications, DMTs were classified into first-line DMTs (glatiramer-acetate, interferons, dimethyl-fumarate, teriflunomide, azathioprine) or second-line DMTs (natalizumab, fingolimod, cladribine, alemtuzumab, ocrelizumab, rituximab); the effect of drugs with a depletive mechanism of action (i.e., ocrelizumab, rituximab, alemtuzumab and cladribine) was further explored comparing this class with not depleting treatments.

Occurrence of adverse events (AEs) and SARS-Cov2 infection following the vaccine administration were also investigated by dedicated phone interviews, and recorded.

\section{Statistical methods}

Baseline characteristics of patients are reported as median and range or as number and frequency, as appropriate. Comparisons between groups were carried out using non-parametric tests (Mann-Whitney test for continuous and Chisquare test for dichotomic variables). Correlations between anti-S antibodies titre and baseline characteristics were explored with Spearman correlations. The statistics software used was SPSS version 25 (Windows); graphing with Origin 2020. A two tailed $p$ value $<0.05$ was considered significant. 


\section{Data availability statement}

Individual de-identified participant data will be shared upon written request.

\section{Results}

\section{Patient characteristics}

One hundred and twenty MS patients were included. Baseline characteristics are summarized in Table 1. Briefly, there were 83 females (69\%); MS form was relapsing-remitting (RR-) in 109 cases (91\%) and secondary-progressive (SP-) in 11 cases. Most of the patients $(112 / 120,93 \%)$ were receiving active treatment at the time of vaccination (Table 2): a I line DMT in $29 / 112$ cases (26\%) and a II line one in the remaining 83 cases (74\%).

\section{Antibody response and DMT at vaccination}

Antibody testing after a single dose was available for $19 / 120$ cases $(16 \%)$. The median titre was $202 \mathrm{BAU} / \mathrm{mL}$ (range $0-1290$ ) and it increased at T2 (median $600 \mathrm{BAU} / \mathrm{mL}$, range 72-2670), $p<0.001$ (Fig. 1a).

Anti-S protein IgG antibody titre above the cut-off at $\mathrm{T} 2$ was detected in 102/120 (85\%) cases; the frequency of responders was lower in MS cases treated with depletive treatments $(17 / 34,50 \%)$ compared to those who received non-depletive treatments $(77 / 78,99 \%), p<0.0001$. At T2, the frequency of responders was $100 \%$ in untreated patients $(n=5)$, and in the group of patients who were receiving a first-line treatment (injectable treatments, dimethyl-fumarate or teriflunomide) or NTZ ( $n=29$ and 35 , respectively). Eleven/12 (92\%) cases receiving FTY and 14/31 (45\%) of cases receiving anti-CD20 antibodies were responders (Fig. 1b).

Details on the remaining patients treated with alemtuzumab $(n=1)$, autologous hematopoietic stem cell transplantation (AHSCT, $n=2)$, cladribine $(n=3)$ or cyclophosphamide $(n=2)$ are reported in Table 3.

In responders, a median antibody titre of $1122 \mathrm{BAU} /$ $\mathrm{mL}$ (range 9.34-9894) was observed, and it was of median $1542 \mathrm{BAU} / \mathrm{mL}$ (range 75-9894) for patients receiving a I line DMT and of median $723 \mathrm{BAU} / \mathrm{mL}$ (range 9-7310) for patients receiving II line DMTs, $p=0.130$ Amongst those receiving a II line DMT, the antibody titre was lower in patients treated with depletive treatments $(27.36 \mathrm{BAU} / \mathrm{mL}$, range 0-3800) compared to those who were not (1047 BAU/ $\mathrm{mL}$, range $0.44-7310), p<0.0001$, and in pwMS treated with fingolimod compared to first-line DMTs, NTZ or no treatment $(p<0.0001)$ (Fig. 2).

\section{Antibody response and baseline blood cell count}

Baseline blood cell count was available for 42/120 (35\%) cases. Thirty-seven/42 (88\%) cases were receiving DMTs at the time of vaccination. The median white blood cell count was 5300 (range 2630-8890). Median baseline white blood cell count did not differ between responders (median 5180, range 2630-8890) and non-responders (median 6070, range 3400-7900), $p=0.564$ (Fig. 3a).

Table 1 Clinical-demographic characteristics of the patient population at the time of SARS-Cov2 vaccine administration

\begin{tabular}{llc}
\hline & Median & $($ Range) \\
\hline Age, years & 42 & $(21-73)$ \\
Disease duration, years & 10 & $(0-38)$ \\
Treatment duration since first DMT, years & 8 & $(0-25)$ \\
Number of previous DMTs & 1 & $(0-5)$ \\
Duration of treatment with the current DMT, months & 36 & $(1-308)$ \\
EDSS & 1.5 & $(0-7.5)$ \\
\hline & Number & $(\%)$ \\
\hline Gender, female & 83 & $(69)$ \\
MS form, RR & 109 & $(91)$ \\
MS form, SP & 11 & $(7)$ \\
On treatment & 112 & $(93)$ \\
Second line DMTs & 83 & $(69)$ \\
Depletive DMTs & a & 34 \\
\hline
\end{tabular}

$D M T$ disease-modifying treatment, $E D S S$ expanded disability status scale, $M S$ multiple sclerosis, $R R$ relapsing-remitting, $S P$ secondary progressive

${ }^{a}$ Depletive DMTs include all the following: rituximab, ocrelizumab, alemtuzumab, cladribine 
Table 2 Disease-modifying treatment received at the time of vaccination

\begin{tabular}{lclll}
\hline & $\begin{array}{c}\text { Number } \\
\text { of cases }\end{array}$ & $(\%)$ & $\begin{array}{l}\text { Median dura- } \\
\text { tion, months }\end{array}$ & (Range) \\
\hline Azathioprine & 1 & $(1)$ & 26 & n.a. \\
Cyclophosphamide & 2 & $(2)$ & 10 & $(6-13)$ \\
Cladribine & 3 & $(3)$ & 17 & $(6-20)$ \\
Fingolimod & 12 & $(11)$ & 59 & $(14-101)$ \\
Glatiramer-acetate & 2 & $(2)$ & 26 & $(5-48)$ \\
Interferons & 9 & $(8)$ & 88 & $(7-151)$ \\
Natalizumab & 35 & $(32)$ & 38 & $(1-130)$ \\
Ocrelizumab & 20 & $(18)$ & 19 & $(2-49)$ \\
Rituximab & 11 & $(10)$ & 39 & $(25-57)$ \\
Tecfidera & 15 & $(14)$ & 38 & $(12-308)$ \\
Teriflunomide & 2 & $(2)$ & 24 & $(18-30)$ \\
\hline
\end{tabular}

No significant correlations were observed between anti$\mathrm{S}$ antibodies titre and baseline white blood cell counts for the 42 evaluable cases. The immunophenotyping did not differ between the two groups (Fig. 3b-e), except for the CD19+ count, which was significantly higher in the responder group (median 139, range $0-1480$ ) than in the non-responder group (median 0 , range $0-7$ ), $p<0.0001$ (Fig. 3f).

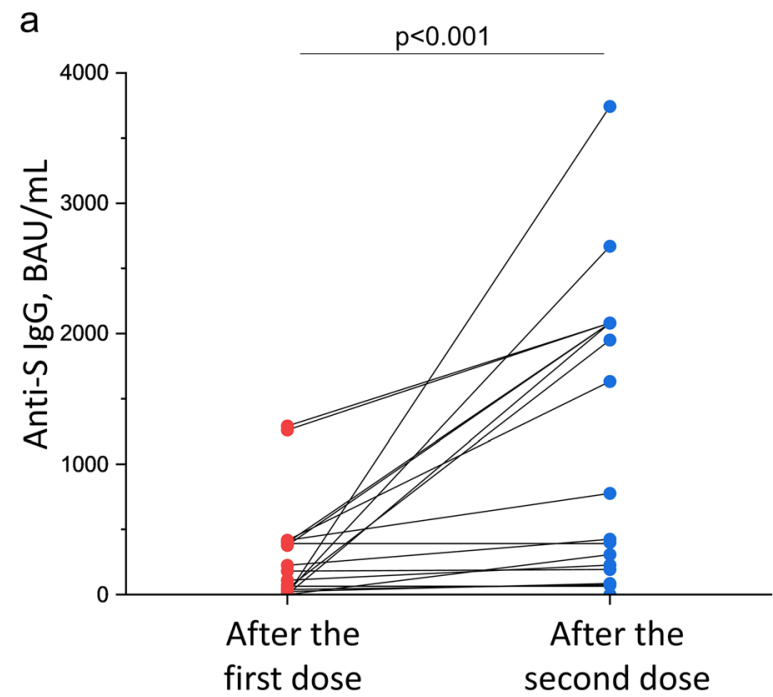

Fig. 1 a Anti-Spike (S) protein IgG antibodies titres in 19 pwMS who were tested both following the first dose and following the second dose of mRNA anti-SARS-Cov2 vaccines. Titres were significantly higher following the second dose than following the first dose $(p<0.0001)$. b Frequency of patients who showed a positive anti-S

\section{Antibody response and clinical-demographic characteristics}

Analysing the whole cohort, no significant correlations were observed between antibody response at $\mathrm{T} 2$ and baseline clinical-demographic characteristics (age and disease duration at vaccination, EDSS).

Correlations were then explored for each DMT group. A significant correlation with age at vaccination was observed in the NTZ group $(R-0.38, p=0.022)$ and patients not treated with depletive treatments $(R-0.25, p=0.028)$. Antibody titre correlated inversely with the duration of treatment with anti-CD20 antibodies $(R-0.93, p<0.0001)$, the number of administrations received $(R-0.59, p=0.001)$ and the time interval between last dose administration and vaccination $(R 0.41, p=0.028)$, (data not shown).

\section{Antibody response and type of mRNA vaccination}

Seventy-eight/120 patients (65\%) received vaccination with Moderna, 40/120 (33\%) with Pfizer and two/120 (2\%) with AstraZeneca. Antibody titre did not differ between patients who received Moderna compared to Pfizer $(p=0.846)$, not even within each DMT group, but the small sample size could have prevented us from finding significant differences (data not shown).

b

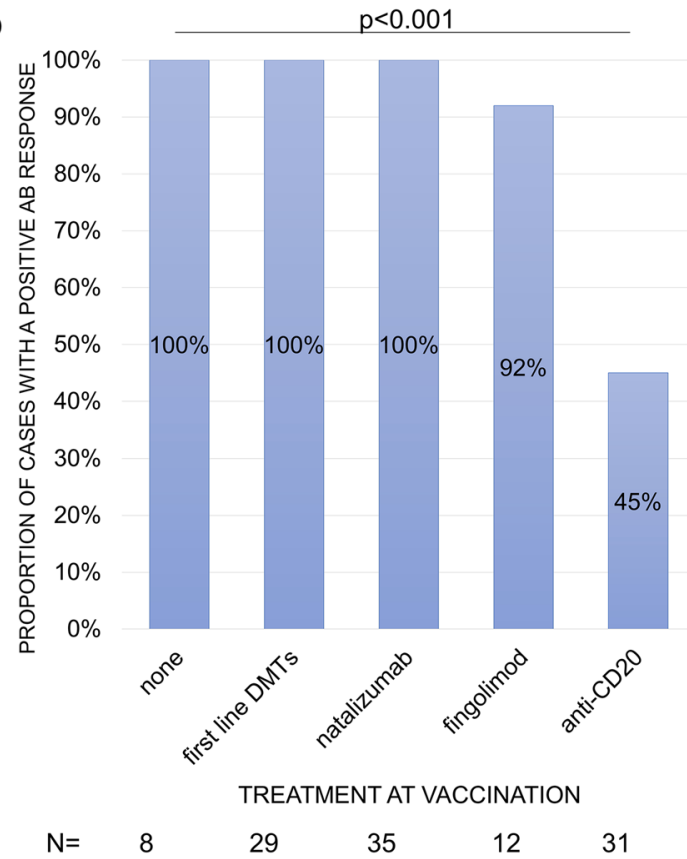

antibodies response according to the disease-modifying treatment (DMTs) received at the time of vaccination. The proportion of cases who showed a positive response was lower amongst pwMS treated with anti-CD20 antibodies compared to those who were receiving different DMTs $(p<0.001)$ 
Table 3 Case series of patients treated with cladribine, AHSCT, alemtuzumab or cyclophosphamide

\begin{tabular}{lllll}
\hline & Cladribine & AHSCT & Alemtuzumab & Cyclophosphamide \\
\hline Number of pwMS & 3 & 2 & 1 & 2 \\
Median age at vaccination (range), years & $34(27-48)$ & $43(43-43)$ & 26 & $46(41-51)$ \\
Median disease duration at vaccination (range), years & $5(0-7)$ & $25(24-26)$ & 4 & $20.5(17-24)$ \\
Median treatment duration since first DMT (range), years & $4(0.5-6)$ & $23(22-24)$ & 3 & $13(10-16)$ \\
Median time since last dose received (range), months & $11(7-15)$ & $60(31-89)$ & 30 & $0.7(0.6-0.8)$ \\
Median number of previous DMTs (range) & $1(0-2)$ & $3(2-3)$ & 0 & $1(1-1)$ \\
Median EDSS (range) & $1.0(0.0-2.5)$ & $2.0(1.0-3.0)$ & 1.0 & $6.25(6.0-6.5)$ \\
Umoral response, $n(\%)$ & $3(100 \%)$ & $2(100 \%)$ & $1(100 \%)$ & $2(100 \%)$ \\
\hline
\end{tabular}

\section{COVID-19 infection}

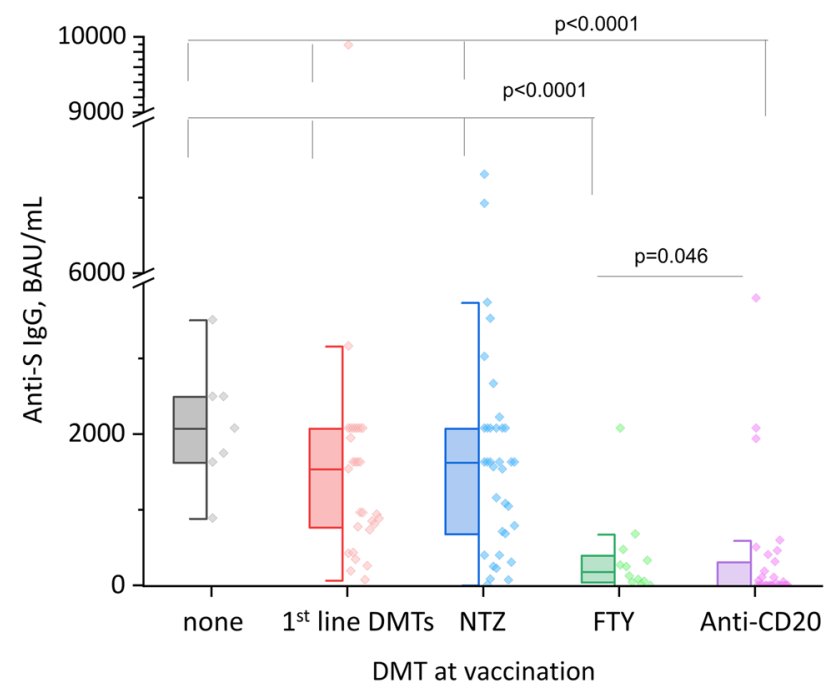

Fig. 2 Anti-Spike protein $\operatorname{IgG}$ antibodies titres in pwMS grouped according to the disease-modifying treatment (DMTs) they were receiving at the time of vaccination. Antibody titre did not differ between patients treated with first-line DMTs, natalizumab (NTZ) or receiving no treatment. Lower titres were observed in pwMS treated with anti-CD20 antibodies and fingolimod compared to the other groups $(p<0.0001)$. The antibody titres below the sensitivity cut-off of the test are reported as 0

\section{Adverse events}

Common adverse events including injection's site pain, fever, and asthenia were reported each by roughly onethird of the patients. Details on the frequency of each adverse event are reported in Fig. 4. Two patients experienced a clinical relapse at week 6 and 12 following the second dose, respectively. One was treated with dimethylfumarate for the last 4 years, while the other patient was treated with AHSCT 8 years before, and was free from therapy since then. They were both treated with high-dose IV methylprednisolone with complete recovery.
Median follow-up after the first dose of vaccine was 5 months (range 2-7). Two patients reported symptomatic COVID-19 over follow-up. One female aged 49 years old experienced fever with respiratory symptoms at month 4 following the completion of the vaccination cycle, requiring access to the emergency department and treatment with antiSARSCov2 antibodies, followed by complete recovery without hospitalisation. At the time of vaccination, this patient was treated with rituximab (starting in 2018) and did not show any humoral response to the vaccination. The other patient is a 37 years old male treated with fingolimod since 2016, without lymphopenia at blood tests taken over the last year; 6 months following the completion of the vaccination cycle (and 1 month after having received the third dose of vaccine) he experienced fever for 3 days and mild respiratory symptoms not requiring hospitalization. Antibody response following the second dose of vaccine was positive with a low titre.

\section{Discussion}

In the last 2 years, the outbreak of the pandemic COVID-19 deeply affected everyday living, and a higher risk of severe infection was first reported in pwMS. Guidelines on DMTs use over the COVID era had changed over time, and uncertainty on a protective vaccine response while receiving definite classes of DMTs had emerged.

A retrospective monocentric study was undertaken to explore the antibody response to anti-SARS-Cov2 vaccines in pwMS receiving various DMTs. One hundred and twenty MS patients were included. Most of the cases were affected by RR-MS and were receiving active treatment at the time of vaccination. An increase in the antibody titre was detected following the second jab of vaccine compared to the sample collected between the two doses, as expected.

A positive anti-S IgG antibody response following completion of the vaccination cycle was detected in $85 \%$ of the 

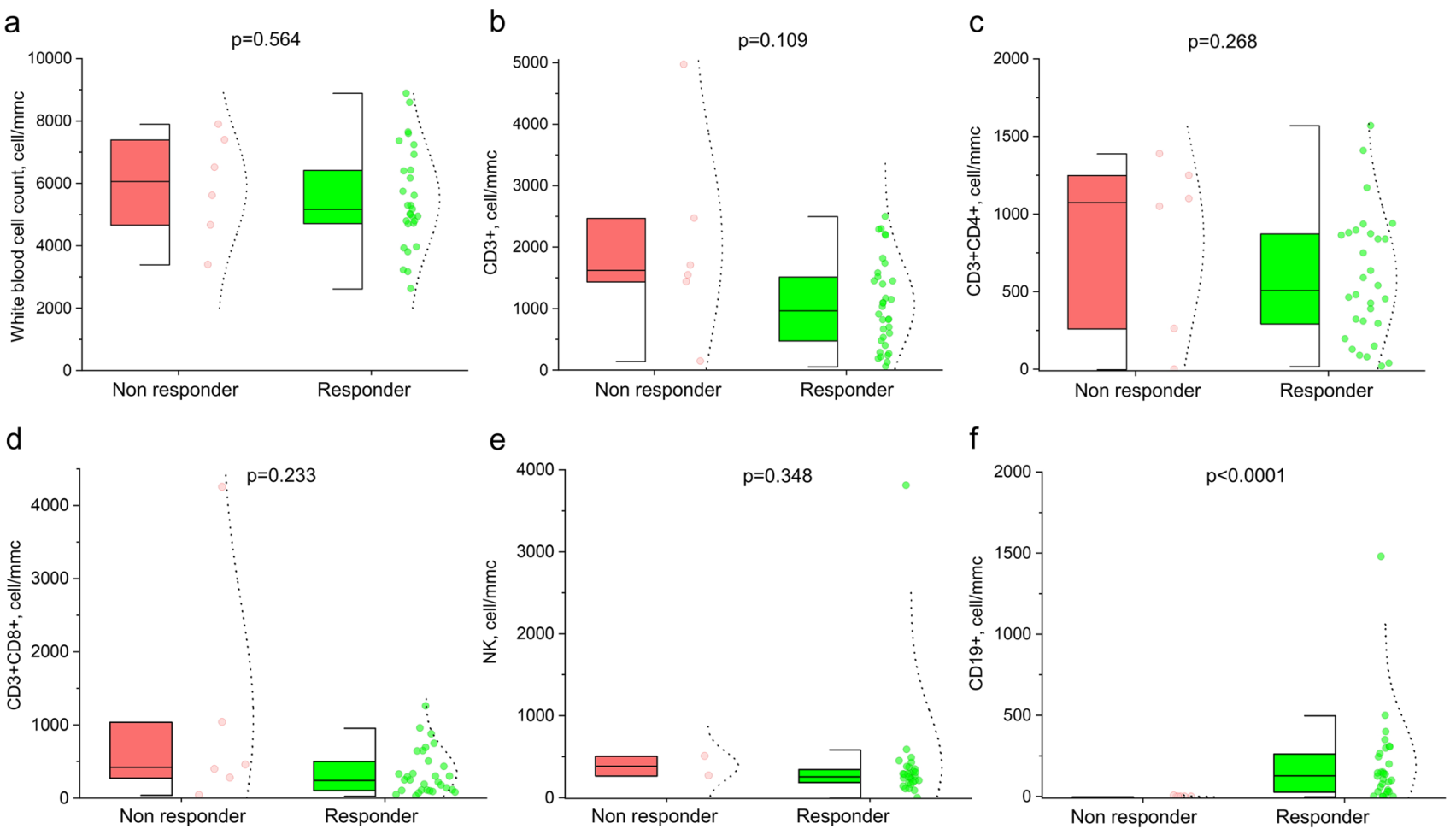

Fig. 3 White blood cell count and immune cell subtypes at baseline of vaccination in pwMS who showed antibody response above (responders) or below the cut-off of detection of the test (nonresponders). CD19+cell count was significantly lower in non-

responders compared to responders $(p<0.0001)$, whereas the remaining cell counts analysed (total white blood cell, CD3+, CD3+CD4+, $\mathrm{CD} 3+\mathrm{CD} 8+, \mathrm{NK})$ did not differ between the groups
Fig. 4 Adverse events reported following anti-SARS-Cov2 vaccination. The proportion of patients who experienced each adverse event is reported for the overall sample $(n=120)$

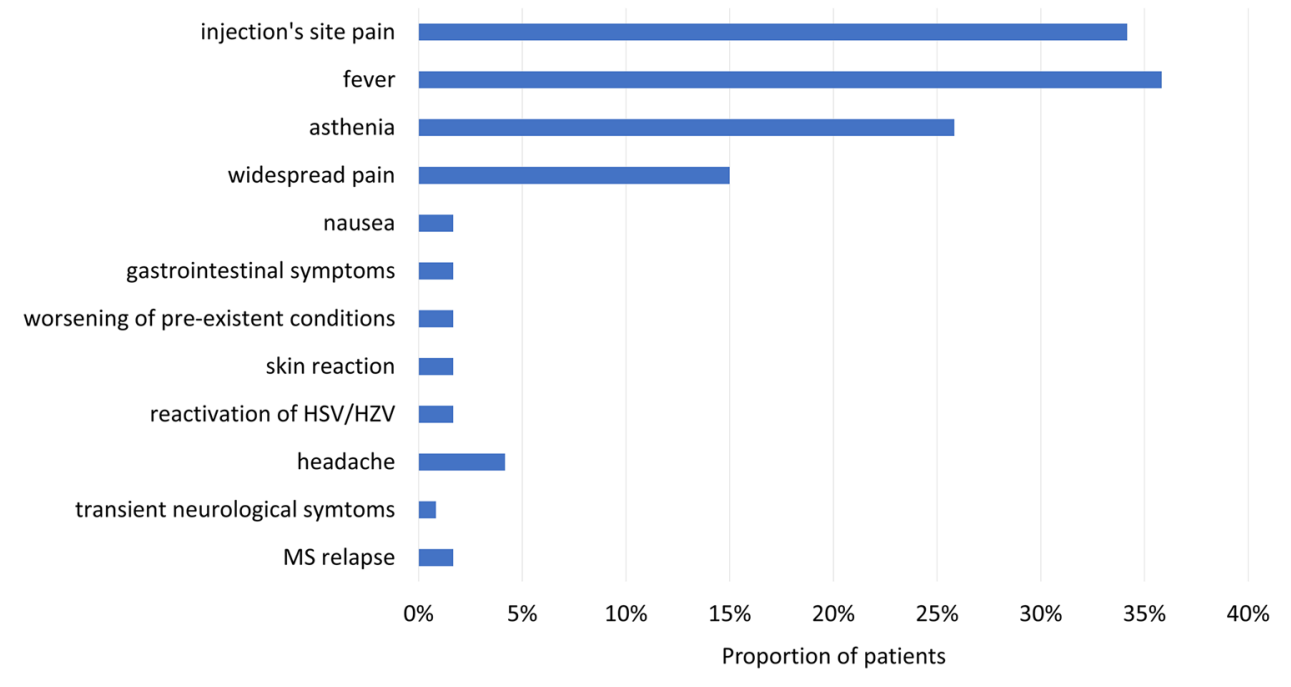

cases. Non-responders were amongst patients treated with FTY (8\%) and anti-CD20 antibodies (55\%). These data are aligned with the literature, reporting a lower response in patients treated with these two classes of DMTs compared to healthy subjects and untreated MS patients or patients receiving other DMTs $[12,14,15]$. Similarly, the odd of developing antibody response following exposure to COVID-19 infection was lower in patients treated with ocrelizumab compared to those who were receiving other DMTs [16].

A few patients received treatment with cladribine, alemtuzumab or AHSCT, showing in all the cases a positive antibody response, but the small number did not allow us to perform any statistical analyses. However, all the cases 
had received the last administration of treatment at least 1 year before vaccination and therefore a positive antibody response was expected according to the known kinetics of immunorepopulation.

Antibody titre showed an inverse correlation with age at vaccination in patients not treated with anti-CD20 antibodies, as previously reported in healthy individuals [17]. This observation might help determine the timing for revaccination in this patient population. For cases treated with anti-CD20 depleting therapies, anti-S antibodies titre was inversely correlated with duration of treatment and with the time interval between the last dose administration and vaccination, these latter data aligned with recent reports in larger cohorts of patients treated with anti-CD20 antibodies [12]. These observations suggest that treatment might not be necessary postponed to allow the completion of the vaccination cycle, as vaccination performed after the first infusion induced a positive antibody response.

Even if the data were available only for a subset of cases, it was interesting, albeit expected, to observe that the CD19+ count was significantly higher in the responder group than in the non-responder group. This suggests that immunophenotyping might be helpful in clinical practice to determine the timing of vaccination in people receiving depletive treatments.

In the present study, the safety profile of the vaccines was aligned with already published data: most of the cases experienced common side effects and relapse of MS was observed in two cases, aligned with previous reports [18, 19]. The occurrence of MS relapse in a patient previously treated with AHSCT and who had been stable for long time up to this event suggests a potential increased risk of disease reactivation in MS patients who are not receiving active treatment at the time of vaccination, and further investigations are needed to ascertain whether this hypothesis is true.

Two cases of symptomatic COVID-19 were reported during the follow-up, but the short duration of observation coupled with the low prevalence of the disease in our geographical area does not allow us to properly estimate the protective effect of vaccines in this sample, however its assessment was beyond the aim of the present study, and it has already been demonstrated.

Our study has several limitations; first of all, its retrospective design did not allow us to collect blood samples and therefore to explore cell-mediated immune-response, an assessment that is not performed by clinical laboratories. This does not allow to provide conclusive data on the actual immunisation status of the patients, given recent evidence on the presence of a positive cell-mediated immune response also in patients who did not show an antibody response after exposure to both the COVID-19 or the mRNA vaccines, and the lack of conclusive data on a correlation between anti-S antibody-titres and protection from COVID-19 [20, 21]. Moreover, for the same reason, the timing of blood sampling was slightly variable between cases and an interval blood sampling between the two doses was not available for all the patients. No differences in antibody response between the two mRNA vaccines were observed, but the relatively small sample size might have prevented us from the detection of significant differences, as higher humoral immunogenicity of the SARSCov2 mRNA-1273 vaccine (Moderna) compared with the BNT162b2 vaccine (Pfizer-BioNTech) has been suggested by other studies $[12,22]$.

\section{Conclusions}

Antibody response to COVID-19 vaccines was detected in most of the pwMS analysed in the present study, but the frequency of responders was lower in pwMS receiving CD20 depleting therapies compared to those treated with other DMTs or untreated ones. Immunophenotyping before vaccine administration might help predict antibody response, especially in older patients who showed lower titres compared to younger ones. However, given recent evidence that effective cell-mediated immune response is elicited by the vaccination also in this MS population, further investigations are needed to properly assess the effectiveness of anti-COVID19 vaccines, especially in those cases who do not show a positive antibody response following vaccination.

Acknowledgements AM reports personal fees from Sanofi, and nonfinancial support from Biogen Idec, Teva, and Novartis, outside the submitted work. CM reports personal fees from Biogen Idec and Novartis, non-financial support fromTeva, Merck, Biogen and Novartis. LM reports non-financial support from Biogen, Novartis, Merck Serono, Genzyme and Teva, outside the submitted work.

Funding The authors received no financial support for the research, authorship, and/or publication of this article.

Data availability Individual de-identified participant data will be shared upon written request.

\section{Declarations}

Conflicts of interest $\mathrm{AB}, \mathrm{LM}, \mathrm{MDC}$, and $\mathrm{AB}$ and $\mathrm{AR}$ have no conflict of interest to declare that are relevant to the content of this article.

Ethical approval The protocol was approved by the local ethics committee (Tuscan region ethics committee for clinical experimentation; approval number 27569; written informed consent was collected according to local regulations. The study has been performed in accordance with the ethical standards laid down in the 1964 Declaration of Helsinki and its later amendments, also in the observation of the specific national laws. 


\section{References}

1. Coronaviridae Study Group of the International Committee on Taxonomy of V (2020) The species severe acute respiratory syndrome-related coronavirus: classifying 2019-nCoV and naming it SARS-CoV-2. Nat Microbiol 5(4):536-544. https://doi.org/10. 1038/s41564-020-0695-Z

2. Baud D, Qi X, Nielsen-Saines K, Musso D, Pomar L, Favre G (2020) Real estimates of mortality following COVID-19 infection. Lancet Infect Dis 20(7):773. https://doi.org/10.1016/S14733099(20)30195-X

3. Thompson AJ, Baranzini SE, Geurts J, Hemmer B, Ciccarelli O (2018) Multiple sclerosis. Lancet 391(10130):1622-1636. https:// doi.org/10.1016/S0140-6736(18)30481-1

4. Montalban X, Gold R, Thompson AJ, Otero-Romero S, Amato MP, Chandraratna D et al (2018) ECTRIMS/EAN guideline on the pharmacological treatment of people with multiple sclerosis. Eur J Neurol 25(2):215-237. https://doi.org/10.1111/ene.13536

5. Louapre C, Collongues N, Stankoff B, Giannesini C, Papeix C, Bensa $C$ et al (2020) Clinical characteristics and outcomes in patients with coronavirus disease 2019 and multiple sclerosis. JAMA Neurol 77(9):1079-1088. https://doi.org/10.1001/jaman eurol.2020.2581

6. Laroni A, Schiavetti I, Sormani MP, Uccelli A (2020) COVID-19 in patients with multiple sclerosis undergoing disease-modifying treatments. Mult Scler. https://doi.org/10.1177/1352458520 971817

7. Hughes R, Pedotti R, Koendgen H (2020) COVID-19 in persons with multiple sclerosis treated with ocrelizumab - a pharmacovigilance case series. Mult Scler Relat Disord 42:102192. https://doi. org/10.1016/j.msard.2020.102192

8. Le TT, Cramer JP, Chen R, Mayhew S (2020) Evolution of the COVID-19 vaccine development landscape. Nat Rev Drug Discov 19(10):667-668. https://doi.org/10.1038/d41573-020-00151-8

9. Jeyanathan M, Afkhami S, Smaill F, Miller MS, Lichty BD, Xing $\mathrm{Z}$ (2020) Immunological considerations for COVID-19 vaccine strategies. Nat Rev Immunol 20(10):615-632. https://doi.org/10. 1038/s41577-020-00434-6

10. Sahin U, Muik A, Derhovanessian E, Vogler I, Kranz LM, Vormehr M et al (2020) COVID-19 vaccine BNT162b1 elicits human antibody and TH1 T cell responses. Nature 586(7830):594-599. https://doi.org/10.1038/s41586-020-2814-7

11. Olberg HK, Cox RJ, Nostbakken JK, Aarseth JH, Vedeler CA, Myhr KM (2014) Immunotherapies influence the influenza vaccination response in multiple sclerosis patients: an explorative study. Mult Scler 20(8):1074-1080. https://doi.org/10.1177/13524 58513513970

12. Sormani MP, Inglese M, Schiavetti I, Carmisciano L, Laroni A, Lapucci C et al (2021) Effect of SARS-CoV-2 mRNA vaccination in MS patients treated with disease modifying therapies. EBioMedicine 72:103581. https://doi.org/10.1016/j.ebiom.2021. 103581

13. Kurtzke JF (1983) Rating neurologic impairment in multiple sclerosis: an expanded disability status scale (EDSS). Neurology 33(11):1444-1452. https://doi.org/10.1212/wnl.33.11.1444

14. Gallo A, Capuano R, Donnarumma G, Bisecco A, Grimaldi E, Conte $\mathrm{M}$ et al (2021) Preliminary evidence of blunted humoral response to SARS-CoV-2 mRNA vaccine in multiple sclerosis patients treated with ocrelizumab. Neurol Sci 42(9):3523-3526. https://doi.org/10.1007/s10072-021-05397-7

15. Achiron A, Mandel M, Dreyer-Alster S, Harari G, Magalashvili D, Sonis P et al (2021) Humoral immune response to COVID-19 mRNA vaccine in patients with multiple sclerosis treated with high-efficacy disease-modifying therapies. Ther Adv Neurol Disord 14:17562864211012836. https://doi.org/10.1177/1756286421 1012835

16. Conte WL (2021) Attenuation of antibody response to SARS$\mathrm{CoV}-2$ infection in patients with multiple sclerosis on ocrelizumab: a case-control study. Mult Scler Relat Disord 52:103014. https://doi.org/10.1016/j.msard.2021.103014

17. Collier DA, Ferreira I, Kotagiri P, Datir RP, Lim EY, Touizer E et al (2021) Age-related immune response heterogeneity to SARSCoV-2 vaccine BNT162b2. Nature 596(7872):417-422. https:// doi.org/10.1038/s41586-021-03739-1

18. Achiron A, Dolev M, Menascu S, Zohar DN, Dreyer-Alster S, Miron S et al (2021) COVID-19 vaccination in patients with multiple sclerosis: what we have learnt by February 2021. Mult Scler 27(6):864-870. https://doi.org/10.1177/13524585211003476

19. Lotan I, Wilf-Yarkoni A, Friedman Y, Stiebel-Kalish H, Steiner I, Hellmann MA (2021) Safety of the BNT162b2 COVID-19 vaccine in multiple sclerosis (MS): early experience from a tertiary MS center in Israel. Eur J Neurol. https://doi.org/10.1111/ene. 15028

20. Iannetta M, Landi D, Cola G, Malagnino V, Teti E, Fraboni D et al (2021) T-cell responses to SARS-CoV-2 in multiple sclerosis patients treated with ocrelizumab healed from COVID-19 with absent or low anti-spike antibody titers. Mult Scler Relat Disord 55:103157. https://doi.org/10.1016/j.msard.2021.103157

21. Apostolidis SA, Kakara M, Painter MM, Goel RR, Mathew D, Lenzi K et al (2021) Cellular and humoral immune responses following SARS-CoV-2 mRNA vaccination in patients with multiple sclerosis on anti-CD20 therapy. Nat Med. https://doi.org/10.1038/ s41591-021-01507-2

22. Steensels D, Pierlet N, Penders J, Mesotten D, Heylen L (2021) Comparison of SARS-CoV-2 antibody response following vaccination with BNT162b2 and mRNA-1273. JAMA. https://doi. org/10.1001/jama.2021.15125 